Rev. SINAPSIS, Vol. 4, No 1, Junio 2014

ISSN $1390-7832$

\title{
Pedagogía del cuidado en la formación profesional de la carrera de Enfermería Pedagogía del cuidado
}

Roberth Zambrano Santos Dr. C. ${ }^{(1)}$

Sonia Patricia Ubillús Saltos. MSc. ${ }^{(2)}$

Margoth Villegas Chiriboga. MSc. ${ }^{(3)}$

Mabel Sánchez Rodríguez. Dra. ${ }^{(4)}$

${ }^{(1)}$ Universidad Estatal del Sur de Manabí. Instituto Tecnológico Superior Portoviejo.

${ }^{(2)}$ Ministerio de Educación. Instituto Tecnológico Superior Portoviejo.

${ }^{(3)}$ Universidad Estatal del Sur de Manabí.

${ }^{(4)}$ Universidad Estatal del Sur de Manabí. Universidad Laica Eloy Alfaro de Manabí.

Contacto: rzambranosantos@yahoo.es

Receptado: 9 /05/2014

Aceptado: 26/05/2014

\section{Resumen}

Este trabajo muestra los fundamentos teóricos de la pedagogía general que sirven de sustentos básicos para entender en que consiste la pedagogía del cuidado, asumiendo como definición a las formas mediante las cuales un profesional de la salud debe entender el cuidado y educar al paciente y a sus familiares en los protocolos de cuidado que debe considerar de acuerdo con la enfermedad que padezca. El mismo se realizó con el propósito de analizar el desempeño de los profesionales graduados en la Universidad Estatal del Sur de Manabí, Ecuador, en enfermería con respecto a las competencias en el cuidado. Los resultados obtenidos demuestran que la agudeza de las patologías en elevado porcentaje se debe a la escasa contribución que los propios pacientes y sus familiares le dan al proceso de prevención de complicaciones y recuperación del buen estado de salud. El objeto de estudio lo constituye la formación profesional, siendo la enfermería la profesión responsable de esta competencia específica.

Palabras clave: pedagogía del cuidado, protocolos de cuidado, diseño curricular, formación profesional, carrera de enfermería. 
Rev. SINAPSIS, Vol. 4, Nº 1, Junio 2014

ISSN $1390-7832$

\title{
Pedagogy of care in the training of nursing career
}

\begin{abstract}
This paper presents the theoretical foundations of general pedagogy that serve as basic principles to understand about what the pedagogy of care means, assuming as the definition, the ways in which a health care professionals must understand the care and educating patients and their families protocols of care that should be considered according to the disease suffered. It was carried out in order to analyze the performance of professional graduated at the "Universidad Estatal del Sur de Manabí”, Ecuador, regarding nursing care skills. The results obtained show that the acuity of the pathologies in high percentage is due to the lack of contribution that the patients themselves and their families give to the process of preventing complications and recovery of good health. The object of study is the vocational training, being the nursing profession responsible for this specific competition.
\end{abstract}

Keywords: pedagogy of care, care protocols, curriculum design, training, nursing specialty.

\section{Introducción}

En la actualidad, un tema bastante controversial en la calidad del trabajo del personal de la salud, sobre todo, en aquellos que tienen relación directa con los pacientes, como son los médicos, paramédicos y enfermeros, es la forma en que orientan el cuidado que requieren los pacientes con enfermedades crónicas para mejorar su calidad de vida.

A través de los tiempos, tanto la enfermería como otras profesiones relacionadas con la salud, han ido desarrollando el contenido de sus funciones como lo han hecho la medicina y demás ramas de la salud, por lo que hoy día su historia puede dar razones de esta evolución, que se ha hecho irreversible para convertirse en profesiones sin perder las originalidades de su esencia: el cuidado. De acuerdo con los historiadores, la enfermería como actividad, ha existido desde el inicio de la humanidad, pues dentro de la especie humana siempre han existido personas incapaces de valerse por sí mismas y siempre se ha visto la necesidad de cuidar de ellas. (León, 2006) 
Rev. SINAPSIS, Vol. 4, No 1, Junio 2014

ISSN $1390-7832$

Partiendo de lo mencionado con anterioridad, surge entonces la necesidad de definir el término cuidado, dentro del marco de las ciencias de la salud, puesto que en la actualidad, éste se utiliza para describir las prácticas del personal médico, sin embargo, es criterio de los autores de este trabajo, que el término es mucho más abarcador, pues de hecho, el empleo de este concepto, se ha convertido en objeto de estudio de muchas de las profesiones antes mencionadas, como la de enfermería.

Otros Autores, (García, y otros, 2011) brindan un concepto bastante profundo y abarcador sobre el término cuidado en el área de enfermería y otras ciencias de la salud, y elaboran el concepto, planteando que como tal, se entiende a la esencia de la disciplina de enfermería y tiene como fin cuidar integralmente al ser humano en condiciones de salud y enfermedad mediante enseñanzas de cuidado, sustentadas en un conocimiento teórico - práctico, científico y humanístico, para la conservación y el mantenimiento de la vida con la mejor calidad posible. Partiendo de este criterio, el cuidado no se limita a determinadas atenciones que requieran los pacientes, mucho menos a precauciones que requiera la interacción con éstos, va mucho más allá, pues también incluye todas las acciones, estrategias, métodos y hábitos, que contribuyan a mejorar la calidad de vida en pacientes con enfermedades crónicas o incurables.

Por su parte, (Contreras, y otros, 2010) son del criterio que cuando se habla de cuidado, es necesario ampliar la esencia del término, para no delimitarlo a la parte instrumental. La acción de cuidar va más allá de lo manual, implica del ser que lo proporciona unas habilidades que forman parte de su estructura psicosocial, la cual se reflejará de manera negativa o positiva en su actuar frente al sujeto de cuidado.

De los conceptos señalados con anterioridad, se puede considerar el cuidado, en toda la extensión de su significado, como la esencia de muchas carreras pertenecientes al sector de la salud, donde la enfermería se destaca considerablemente. Poblete y Valenzuela, sostienen que el cuidado es la esencia de Enfermería, y que sus componentes deberían estar constituidos por acciones transpersonales e intersubjetivas para proteger, mejorar y preservar la humanidad ayudando a la persona a hallar un significado a la enfermedad, sufrimiento, dolor y existencia y ayudar a otro a adquirir autocontrol, autoconocimiento y autocuración. (Poblete Troncoso \& Valenzuela Zuazo, 2007) 
Rev. SINAPSIS, Vol. 4, No 1, Junio 2014

ISSN $1390-7832$

Actualmente la enseñanza de carreras relacionadas con las ciencias de la salud, como es la Enfermería, es bastante común en casi todas las regiones del planeta, sin embargo, es frecuente y común que se descuide la enseñanza o pedagogía del cuidado de los pacientes, hecho que resulta inaceptable, dada la importancia que éste concepto tiene en las carreras tratadas.

Entonces puede verse el concepto de cuidado, como una habilidad o una competencia que resulta imprescindible formar en profesionales de las ciencias de la salud, de las cuales, no se puede escindir, sin embargo, no siempre sucede de este modo.

Partiendo de estos criterios, este trabajo, se propone como objetivo general, analizar el desempeño de los profesionales graduados en la Universidad Estatal del Sur de Manabí en enfermería con respecto a las competencias en el cuidado; y como objetivos específicos se establecen: 1) Identificar las áreas de los hospitales en donde son asignados los profesionales en enfermería graduados en la UNESUM durante su año rural. 2) Determinar el nivel de cumplimiento de las cinco características de cuidar de Roach (1997). 3) Analizar el grado de dominio de las habilidades del cuidado en los graduados de enfermería de la UNESUM; y, 4) Evaluar el cumplimiento de las competencias de la carrera de enfermería de sus graduados.

\section{Materiales y métodos}

Para identificar el concepto de la pedagogía del cuidado se realizó un estudio cualitativo y así comprender aquello que se conoce y que tiene significado solamente en un contexto dado. El método hermenéutico sirvió de marco referencial para la recopilación de la información secundaria.

Se trabajó con toda la población correspondiente a la totalidad de graduados de la carrera en la UNESUM, la cual está formada por un total de 67 encuestados, quienes se desempeñan actualmente como profesionales en la carrera estudiada. En cuanto a los criterios de inclusión se respetó el género y la interculturalidad.

El control de calidad de la información obtenida y la ubicación de los encuestados, se efectuó escogiendo a los graduados de licenciados en enfermería de la Universidad Estatal del Sur de Manabí, que hicieron su año rural, considerándose a éstos porque han trabajado con situaciones 
Rev. SINAPSIS, Vol. 4, No 1, Junio 2014

de al menos, más de un tipo de cuidado, durante uno o dos años, además, cuentan con un bagaje de experiencia y comprensión intuitiva de cada situación y tienen en cuenta un gran número de diagnósticos y soluciones, y sobre todo porque con ellos se trabajó en su formación profesional la pedagogía del cuidado.

Los datos se organizaron empleando el análisis de contenido de manera manual en las siguientes etapas: 1) pre análisis; 2) exploración del material; 3) reagrupamiento de categorías semánticas; 4) categorías temáticas; y 5) interpretación.

Para presentar los datos, se utilizaron los componentes que constituyen las categorías sustantivas, constituidas por: áreas de trabajo de los enfermeros; características de cuidar; las competencias profesionales de enfermería; y el fundamento de las habilidades del cuidado.

El aspecto ético de la investigación se fundamentó en lo que establece el artículo 146 del Código Orgánico Integral Penal, que hace referencia al "manejo ético del cuidado". (Asamblea Nacional Constituyente, 2014)

\section{Resultados}

Con el propósito de evaluar la calidad de los desempeños profesionales de los graduados en enfermería de la UNESUM, se identificaron las diferentes áreas donde desarrollaron el año rural los mencionados profesionales, entendiéndose que en cada área se desempeñan diferentes tipos de cuidados. La tabla 1 muestra las áreas en las cuales laboraron durante su año de internado rural los egresados, y se demuestra que existe equidad en la distribución de los mismos, pues no existen áreas específicas preferenciales para dirigir los profesionales en enfermería graduados en la UNEUSM.

Tabla 1. Áreas de trabajo de Enfermeros y Enfermeras en su año rural.

\begin{tabular}{cccc}
\hline Áreas de trabajo & Cantidad & Hombres & Mujeres \\
\hline Pediatría & 14 & 0 & 14 \\
\hline
\end{tabular}


Rev. SINAPSIS, Vol. 4, No 1, Junio 2014

Emergencias

11

2

9

Medicina interna

9

0

9

Ginecología

13

0

13

Centro Quirúrgico

8

1

7

Otras áreas

12

2

10

\section{TOTAL}

La tabla 2, muestra los resultados del cumplimiento de las características de cuidar que presenta Simone Roach, por parte de los egresados de la carrera de enfermería.

En esta tabla se aprecia, que la característica compasión es la que más dificultades crea a los enfermeros, aunque el $43 \%$, es decir, casi la mitad; tiene desarrollada esta característica en el nivel alto, existe un mayor rechazo al término compasión que a su significado.

La confianza es muy necesaria para poder establecer una relación significativa con los pacientes y ésta se desarrolla en las relaciones entre enfermeros y pacientes, pues en no pocas ocasiones, corresponde a los primeros explicar detalladamente el significado de su enfermedad a los segundos, también el enfermo nota de inmediato las habilidades procedimentales de su enfermero. Esta característica demuestra mejores resultados en los egresados de enfermería ya que el $45 \%$ genera confianza en un nivel alto. Cuando los enfermeros no se sienten seguros ni competentes con los cuidados que están prestando, y con las informaciones que brindan, los enfermos se percatan de esta situación con facilidad y pierden rápidamente la confianza, de ahí la necesidad de informar a éstos de su situación, sin que se muestre una falsa competencia que el 
Rev. SINAPSIS, Vol. 4, Nº 1, Junio 2014

enfermo rápidamente capta, de igual manera, no es menos importante, que el enfermero inspire confianza, mediante sus habilidades y acertados procedimientos.

Ante la competencia, los participantes de la investigación, mostraron estar de acuerdo y la mayoría coincidió en que ésta no era un elemento que les creara problemas a nivel profesional. El $35 \%$ se ubicó en un nivel alto de competitividad, el 36\% en nivel medio y el $29 \%$ en nivel bajo. Estos Remarcaron que para lograr la competencia deseada, deben actualizarse constantemente ante los nuevos conocimientos de la ciencia y no es suficiente el mostrar una buena voluntad. Así mismo indicaron la existencia de problemas en poder ejercer plenamente sus competencias por definición de roles, niveles jerárquicos o funcionalidades hospitalarias. A pesar de que han adquirido una mayor autonomía respecto al poder médico, continúan presentándoseles problemas con el estamento médico.

Tampoco se evidenciaron mayores problemas en cuanto a la conciencia, ya que el $40 \%$ se ubica en un nivel alto, el $34 \%$ en nivel medio y $26 \%$ en nivel bajo. Se resaltaron las dificultades que se presentan a los enfermeros cuando en conciencia no están de acuerdo con los valores que defiende el paciente.

El compromiso se abordó desde el ámbito profesional, como una obligación moral de efectuar las acciones de cuidar de la mejor manera posible. EL 44\% mostró un alto nivel de desarrollo de esta característica, mientras que $37 \%$ se ubican en nivel medio y $19 \%$ en nivel bajo.

Tabla 2. Las cinco características de cuidar de Simone Roach

\begin{tabular}{cccc}
\hline Características & Alto & Medio & Bajo \\
\hline Compasión & $43 \%$ & $31 \%$ & $26 \%$ \\
Confianza & $45 \%$ & $28 \%$ & $27 \%$ \\
\hline
\end{tabular}


Rev. SINAPSIS, Vol. 4, N 1, Junio 2014

Competencia

Conciencia

Compromiso
$35 \%$

$40 \%$

$44 \%$
$36 \%$

$34 \%$

$26 \%$

$29 \%$

$37 \%$

$19 \%$

Para identificar el dominio de las habilidades del cuidado, se analizaron las dimensiones: conocimiento, paciencia y práctica de valores. Cada uno de estos elementos se investigó, y se detectó que la dimensión paciencia se ubica en la de mayor desarrollo, con un $47 \%$, elemento importante en la formación de cualquier enfermero; este resultado guarda relación tanto con las condiciones socioculturales de la población, como con el contexto de desarrollo y la formación sociocultural de ésta. La tabla 3 muestra los resultados del nivel de dominio de las habilidades del cuidado en la escala alto, medio y bajo.

Tabla 3. Dominio de las habilidades del cuidado

$\begin{array}{llll}\text { Dimensiones } & \text { Alto } & \text { Medio }\end{array}$

$\begin{array}{llll}\text { Conocimientos } & 36 \% & 36 \% & 28 \%\end{array}$
Paciencia
$47 \%$
$32 \%$
$21 \%$

Práctica de valores

$35 \%$

$36 \%$

$29 \%$

El diseño curricular de la carrera de enfermería tiene establecidas las competencias que deben desarrollar los profesionales en enfermería en su proceso de formación. La investigación hizo una evaluación del grado de cumplimiento de las cinco competencias claramente identificadas, sobre las cuales se evidenció las que tienen mayor nivel de desarrollo, y se determinó que la quinta 
Rev. SINAPSIS, Vol. 4, No 1, Junio 2014

ISSN $1390-7832$

competencia que indica: Intervenir con acciones preventivas durante el proceso saludenfermedad, así como en casos de urgencias y/o desastres, es la que está en el nivel más alto de desarrollo (38\%); la primera y cuarta competencias que respectivamente manifiestan: Manejar y usar guías para la valoración de enfermería, incluyendo la aplicación de estrategias y técnicas de entrevistas y visitas tanto hospitalarias como no hospitalarias; y, Diseñar, planear, ejecutar y evaluar intervenciones de enfermería en los diferentes escenarios del cuidado a la salud, se ubican en un término medio en cuanto a nivel de dominio (41\% y 37\% respectivamente); mientras que la competencia de más bajo desarrollo es la tercera, es decir, Manejar la información para la organización, registro e interpretación con el fin de jerarquizar las necesidades y derivar el plan de cuidados, con el 34\%.

En la tabla 4, se presentan los resultados del nivel de dominios de las competencias en los enfermeros graduados en la UNESUM.

Tabla 4. Nivel de cumplimiento de las competencias de la carrera de enfermería.

\section{Competencias específicas}

\section{Nivel de dominios}

\begin{tabular}{l|l}
\hline Alto & Medio
\end{tabular}

Manejar y usar guías para la valoración de enfermería, incluyendo la aplicación de estrategias y técnicas de entrevistas y visitas $31 \%$ $41 \%$ $28 \%$ tanto hospitalarias como no hospitalarias

Observar, escuchar, explorar, inferir y elaborar $36 \%$ $35 \%$ $29 \%$ un diagnóstico de enfermería

Manejar la información para la organización, registro e interpretación con el fin de jerarquizar las necesidades y derivar el plan de
$36 \%$ $34 \%$ 
Rev. SINAPSIS, Vol. 4, № 1, Junio 2014

cuidados

Diseñar, planear, ejecutar y evaluar

$34 \%$

$37 \%$

$29 \%$

intervenciones de enfermería en los diferentes

escenarios del cuidado a la salud

Intervenir con acciones preventivas durante el

$38 \%$

$35 \%$

$27 \%$

proceso salud-enfermedad, así como en casos

de urgencias y/o desastres

Discusión y análisis de los resultados

Partiendo de la valoración de los resultados, se puede entender que el concepto de cuidado es parte del lenguaje de los enfermeros, quienes reconocen y dan significado al término de cuidado como la esencia de la profesión. Son varios los autores que a nivel mundial han tratado este término, por ejemplo, Poblete, afirma que el cuidado es la esencia de la enfermería, mientras que Watson, sostiene que el mismo es el que está constituido por el conjunto de acciones transpersonales e intersubjetivas para proteger, mejorar y preservar la humanidad ayudando a la persona a hallar un significado a la enfermedad, sufrimiento, dolor y existencia y ayudar a otro a adquirir autocontrol, autoconocimiento y autocuración. (Watson J. , 1985), (Watson J., 2005)

Otros autores, consideran que el concepto del cuidado, dentro de la profesión de la enfermería, es el fenómeno central dentro de la disciplina de la profesión. (Herrera \& Riviera, 2006)

Apoyado en lo expresado anteriormente, para proporcionar un Cuidado Profesional se requiere de conocimientos, actitudes y destrezas que sólo se adquieren con el estudio profundo y sistemático de la sociedad, del hombre, la salud y la propia disciplina de enfermería (García H. M., 2011). 
Rev. SINAPSIS, Vol. 4, No 1, Junio 2014

También es importante señalar, que para brindar un cuidado óptimo y profesional, no bastan los elementos mencionados por el autor citado con anterioridad, pues también se precisa de aptitudes por parte del personal encargado del cuidado del paciente, parte de las cuales, el enfermero o cualquier persona poseen de manera innata, pero otra, y por lo general, la mayor, se puede desarrollar mediante un adecuado trabajo educativo en la formación axiológica, psicológica, moral, espiritual y ética de los futuros enfermeros, sin embargo, este proceso educativo, no es una tarea fácil de asumir, y en muchas instituciones formadoras o educativas, se ha ido dejando de lado, quedando este tipo de enseñanza incompleta, e incluso, inabordada, de ahí que no sea difícil encontrar en los hospitales modernos, policlínicas o centros de salud, enfermeros competentes desde el punto de vista técnico, pero incapaces de dispensar a los pacientes el cuidado humano, comprensivo, compasivo e incluso afectivo, que la mayoría de los seres humanos necesitan, para acelerar el proceso de curación y mejorar su calidad de vida. Con relación a esto, Caro es del criterio que La Enfermería como disciplina, debe orientar sus acciones al cuidado integral de la vida y de la salud del ser humano, bien a individuos o bien a colectivos, y esto solamente es posible lograrlo, mediante un cuidado integral y completo. (Caro, 2009).

Autores como Herrera y Rivera, sostienen que el cuidado comprensivo dinamiza este proceso y estimula la movilización del sujeto desde la parálisis que provoca la condición desfavorable de salud, hacia acciones que abren nuevas posibilidades para la salud. (Herrera \& Riviera, 2006)

El proceso de enseñanza aprendizaje de la carrera de Enfermería en la UNESUM, parte de las ideas de los autores mencionados, e incluye además las de Fawsett, quien sostiene que dos elementos esenciales conforman la carrera de Enfermería, uno es el profesional, que la integra con otras disciplinas de salud, facilitándoles el desarrollo de acciones que mejoran la calidad de vida y dan respuestas a las necesidades sociales; el otro elemento es el disciplinar, que representa el cuerpo de conocimientos relacionados con el estudio del cuidado, esencia misma de la profesión y que está condicionado por la sociedad, ante sus necesidades específicas de atención. (Fawsett, 2005).

La carrera de enfermería, que se estudia en la Universidad Estatal del Sur de Manabí, está formada por 64 asignaturas, de éstas en algunas de ellas, entre las que se destacan, Pedagogía y Didáctica, Psicología Social y Evolutiva. Investigación en Salud, Ética Profesional y Bio-Ética, realizan un trabajo bastante profundo en lo relacionado con el desarrollo en los estudiantes de las 
Rev. SINAPSIS, Vol. 4, No 1, Junio 2014

ISSN $1390-7832$

características del cuidado, a través de la formación de valores y principios en los estudiantes, lo cual contribuye de este modo a una formación más integral y universal del alumnado.

A través del desarrollo del currículo de las asignaturas antes citadas y por sus características, dentro de éstas se trabajan valores como: honestidad, solidaridad, respeto, responsabilidad; entre otros.

Esto se hace partiendo del criterio de que el enfermero debe estar preparado para dispensar un cuidado integro, completo, que bajo ningún concepto pueda limitarse a actividades procedimentales solamente, y sobre todo, que debe estar formado por las diferentes características del cuidado, formuladas por Simmone Roach.

Con respecto a lo antes mencionado, hay que señalar que los autores de este artículo, coinciden con el creiterio de Zarate Grajales, quién expone que la enfermería se ocupa más allá del acto de cuidar que une el "qué" del cuidado y el "cómo" de la interacción persona-enfermera, implica crear un cuidado que recurra a diversos procesos: la reflexión, la integración de creencias y valores, el análisis crítico, la aplicación de conocimientos, el juicio clínico, la intuición, la organización de los recursos y la evaluación de la calidad de las intervenciones. Desde esta perspectiva se trata de un cuidado innovador que une la ciencia y el arte de enfermería y que se centra en la persona, la cual en continua interacción con su entorno, vive experiencias de salud. (Zarate, 2004)

El sujeto del cuidado, indiscutiblemente, es el ser humano, en coinciden todos los investigadores en salud. Los enfermeros, como profesionales de la disciplina, igualmente prestan sus servicios a grupos humanos (familia y comunidad). El cuidado de la salud, desde la perspectiva de la formación de los enfermeros en la UNESUM, se enfoca partiendo de una visión global del hombre, considerando los aspectos fisiológicos, psicológicos y sociales que están presentes en todas y cada una de las personas. Con ello los autores de este trabajo se identifican por completo con el concepto de cuidado dado por Poblete y Valenzuela.

Sin embargo, es importante también mencionar que bajo ningún concepto puede descuidarse, la formación científica, procedimental y técnica, pues una forma integral de cuidado, no justifica en ninguna medida, que el aprendizaje laico, técnico y práctico - procedimental de los enfermeros, 
Rev. SINAPSIS, Vol. 4, No 1, Junio 2014

ISSN $1390-7832$

se vea disminuido o afectado: un tipo de contenidos y aprendizajes, no implica, el descuido o la reducción de otras.

\section{Conclusiones}

Luego de la revisión bibliográfica y el análisis de las encuestas realizadas, se puede evidenciar que el cuidado presenta una gran complejidad cuando es aplicado en enfermería. La teoría como la práctica tiene siempre un trasfondo cultural, por lo que debe ser analizada teniendo en cuenta las diferentes perspectivas culturales. Partiendo de estas dos premisas, las conclusiones del presente trabajo se pueden sintetizar en:

La metodología de trabajo de la pedagogía del cuidado desde el currículo de la carrera de enfermería ha dado resultados satisfactorios en la formación de los enfermeros, ya que se evidencia el cumplimiento de las competencias establecidas, muestran un aceptable dominio de las habilidades de cuidar y sobre todo un equilibrio significativo de las características de cuidar de Roach. Además es importante aclarar que si bien es cierto que desde la perspectiva del cuidar son las emociones y no la razón las que hacen pensar en las necesidades de los demás, también lo es el que la respuesta moral no es simplemente emocional, el cuidado tiene una función cognitiva que hay que tener en cuenta ya que permitirá conocer y entender las necesidades para poder respetarlas. También hay que señalar, el aprendizaje sobre un cuidado integral que se trata de lograr en la Universidad Estatal del Sur de Manabí, Ecuador.

\section{Bibliografía}

1. Asamblea Nacional Constituyente. (2014). Código Orgánico Integral Penal. Quito: Reistro Oficial No. 180.

2. Caro, S. (2009). Enfermería: Integración del cuidado y el amor. Salud Uninorte, 25(1), 172 - 178.

3. Contreras, A., Contreras, A., Díaz, M. E., Hernández, M., Muñoz, N., \& Ricardo, M. C. (2010). Habilidad de cuidado de los profesionales de enfermería de una. Universidad Libre. Sección Pediatría, 17 - 23. Obtenido de http://dialnet.unirioja.es/servlet/articulo?codigo=3785199

4. Fawsett, J. (2005). Criteria for Evaluation of theory. Nursing Science Quarterly, 18(2), 131 - 135. 
Rev. SINAPSIS, Vol. 4, No 1, Junio 2014

ISSN 1390 - 7832

5. García, H. M. (2011). Construcción emergente del concepto: Cuidado Profesional de Enfermería. Florianópolis, 74-80.

6. García, H. M., Cárdenas Becerril, L., Arana Gómez, B., Monroy Rojas, A., Hernández Ortega, Y., \& Salvador Martínez, C. (2011). CONSTRUCCIÓN EMERGENTE DEL CONCEPTO:

CUIDADO PROFESIONAL DE ENFERMERÍA. Texto Contexto Enferm, Florianópolis, 20, 4480. Obtenido de http://www.scielo.br/pdf/tce/v20nspe/v20nspea09.pdf

7. Herrera, L. M., \& Riviera, M. S. (2006). FUNDAMENTOS FENOMENOLÓGICOS PARA UN CUIDADO COMPRENSIVO DE ENFERMERÍA. Texto Contexto Emfermería Florianópolis, 158 - 63. Obtenido de http://www.scielo.br/pdf/tce/v15nspe/v15nspea19.pdf

8. León, R. C. (2006). Enfermería ciencia y arte del cuidado. Rev Cubana Enfermería, 22(4). Obtenido de http://www.bvs.sld.cu/revistas/enf/vol22_4_06/enf07406.htm

9. Poblete Troncoso, M., \& Valenzuela Zuazo, S. (2007). Cuidado humanizado: un desafio para las enfermeras en los. Acta Paul Enferme, 20(4), 499-503. Obtenido de http://www.scielo.br/pdf/ape/v20n4/18.pdf

10. Roach, M. (1997). Caring from the heart: The convergence of caring and spirituality. Paulist Press.

11. Watson, J. (1985). Nursing: the philosophy and science of caring. Colorado: Colorado Associated University Press.

12. Watson, J. (2005). Caring science as sacred science. Philadelphia: F.A. Davis Company.

13. Zarate, G. R. (2004). La Gestión del Cuidado de Enfermería. Index de Enfermería, 13(44-45). Obtenido de http://dx.doi.org/10.4321/S1132-12962004000100009 